\title{
Differential Expression of microRNAs in Medulloblastoma and the Potential Functional Consequences
}

\author{
Yuting ZHANG ${ }^{1,2}$, Lusheng $\mathrm{LI}^{3}$, Ping $\mathrm{LIANG}^{3}$, Xuan ZHAI ${ }^{3}$, Yingliang $\mathrm{LI}^{3}$, Yudong ZHOU ${ }^{3}$ \\ ${ }^{1}$ Children's Hospital of Chongqing Medical University, Ministry of Education Key Laboratory of Child Development and Disorders, \\ Chongqing, China \\ ${ }^{2}$ China International Science and Technology Cooperation Base of Child Development and Critical Disorders, Department of Radiology, \\ Chongqing, China \\ ${ }^{3}$ Children's Hospital of Chongqing Medical University, Chongqing Key Laboratory of Pediatrics, Department of Neurosurgery, Chongqing, China
}

\section{ABSTRACT}

AIM: To identify differentially expressed microRNAs in medulloblastoma, and to investigate their biological function.

MATERIAL and METHODS: Differentially expressed microRNAs were identified using gene chips, and significantly different microRNAs were selected for verification using real time quantitative PCR. Potential target genes and their biological pathways were predicted by bioinformatics software.

RESULTS: Our analysis identified two microRNAs, hsa-miR-208a-3p and hsa-miR-1207-5p, which were significantly downregulated in medulloblastoma. Bioinformatics analysis identified potential target genes in the Wnt and MAPK signaling pathways, including NLK, RAPGEF2, CACNA2D1, DUSP3, MAPK8IP3.

CONCLUSION: Downregulation of hsa-miR-208a-3p and hsa-miR-1207-5p may be involved in the occurrence of medulloblastoma, through modulations of the Wnt and MAPK signaling pathways.

KEYWORDS: Medulloblastoma, hsa-miR-208a-3p, hsa-miR-1207-5p, Target gene, Wnt signaling pathway, MAPK signaling pathway

\section{INTRODUCTION}

$\mathrm{M}$ edulloblastoma is a brain tumor that occurs with high incidence, accounting for $10 \%-20 \%$ of all central nervous system tumors in children (5). MicroRNAs (miRNAs) are highly conserved noncoding RNA, which have been shown to inhibit translation, control mRNA shear, promote $\mathrm{mRNA}$ degradation and regulate the expression of target genes. miRNAs have been linked to the occurrence and development of tumors $(2,8)$. In medulloblastoma, miRNA also plays an important role; previous research has shown that miR124a, miR-9, and miR-125a were significantly downregulated in medulloblastoma (4). Because of the complex miRNA regulatory network, through collaboration among multiple miRNAs, high throughput chip hybridization technology seemed a suitable approach for screening tumors to identify differentially expressed miRNAs. At present, the available miRNA chip cover all the miRNA expressed in all species. This study used miRNA chip screening to identify differentially expressed miRNAs in medulloblastoma, and verified the interest of these miRNAs using real time quantitative PCR. We also explored the potential function of the miRNAs using bioinformatics methods, predicting the potential target genes and enriched biological pathways. This gave some insight into the possible biological function of the differentially expressed miRNAs in medulloblastoma. 


\section{MATERIAL and METHODS}

\section{Samples}

The specimens used for these studies included three cases of tumor and three samples of corresponding neighboring tissue; tissue adjacent to the tumor was used as the negative control. Tissues were collected after informed consent from patients with medulloblastoma being treated by the neurosurgery department of Children's Hospital, Chongqing Medical University. Samples were stored in liquid nitrogen before RNA extraction. The patients were aged 1 to 6 years old, and included two males and one female.

\section{miRNA microarray}

miRNA slides were prepared as described by Zhang et al. (19). The seventh generation miRCURYTM LNA Array (v.18.0, Exiqon) was used and contains 3100 capture probes that cover all human, mouse and rat microRNAs annotated in miRBase 18.0, all viral microRNAs related to these species, and capture probes for 25 miRPlus $^{\mathrm{TM}}$ human microRNAs.

Total RNA was isolated using TRIzol (Invitrogen) and the miRNeasy Mini Kit (QIAGEN) per the manufacturers' instructions; we recovered miRNAs. RNA quantity and quality were determined using a NanoDrop ND-1000 spectrophotometer; integrity of RNA was determined by gel electrophoresis. Isolated miRNAs were labeled using the miRCURY'M Hy3 ${ }^{\mathrm{TM}} / \mathrm{Hy} 5^{\mathrm{TM}}$ Power Kit (Exiqon, Vedbaek, Denmark) per the manufacturer's instructions. Each sample $(1 \mu \mathrm{g})$ was $3^{\prime}$-end-labeled with a fluorescent $\mathrm{Hy}^{\mathrm{TM}}$ label using T4 RNA ligase: $2.0 \mu \mathrm{L}$ of RNA solution was mixed with 1.0 $\mu \mathrm{L}$ of CIP buffer and CIP (Exiqon), incubated at $37^{\circ} \mathrm{C}$ for 30 minutes, and then the reaction was terminated by heating at $95^{\circ} \mathrm{C}$ for 5 minutes. Next, $3.0 \mu \mathrm{L}$ of labeling buffer, $1.5 \mu \mathrm{L}$ of $\mathrm{Hy} 3,2.0 \mu \mathrm{L}$ of dimethylsulfoxide, and $2.0 \mu \mathrm{L}$ of labeling enzyme were added and the mixture incubated at $16^{\circ} \mathrm{C}$ for 1 hour. The reaction was terminated by heating to $65^{\circ} \mathrm{C}$ for 15 minutes.

Next, the $\mathrm{Hy}^{\mathrm{TM}}$-labeled samples were hybridized with the miRCURYTM LNA Array per the user manual. The $25-\mu \mathrm{L}$ mixture from $\mathrm{Hy} 3$ labeling was mixed with hybridization buffer $(25 \mu \mathrm{L})$ and denatured at $95^{\circ} \mathrm{C}$ for 2 minutes, then cooled on ice for 2 minutes. The labeled probe was incubated with the microarray at $56^{\circ} \mathrm{C}$ for $16-20$ hours in a 12-Bay Hybridization System (Hybridization System-Nimblegen Systems, Inc., Madison, WI, USA). Afterwards, the slides were washed several times with wash buffer (Exiqon), dried by centrifugation at 400 rpm for 5 minutes, and scanned using an Axon GenePix 4000B microarray scanner (Axon Instruments, Foster City, CA, USA).

\section{Data Analysis}

For grid alignment and data extraction, scanned images were imported into GenePix Pro 6.0 software (Axon). Expression data were normalized using median normalization. Replicated miRNAs were averaged and miRNAs with intensity $\geq 30$ in all samples were used for calculating the normalization factor. MiRNAs with significantly different expression were identified by volcano plot. R Script was used for hierarchical clustering.

\section{Bioinformatics Analysis}

We used the Targetscan, microRNA.ORG and miRDBA databases to identify target genes shared across the three databases for pathway analysis, using all pathways in the genetic information of the KEGG and BIOCARTA. Depending on the known function of the target genes, we used Gene Ontology Analysis to calculate how many target genes were identified, obtain the target genes GO annotation clustering and investigate enrichment of the biological pathways.

\section{Real time Quantitative PCR}

We reverse transcribed RNA into cDNA by real time PCR to detect the expression of target genes, using expression of the $\mathrm{U} 6$ gene as an internal reference. The PCR reaction mix included $1 \mu \mathrm{l} \mathrm{CDNA}, 10 \mu \mathrm{l}$ SYBR premix Taq, $0.5 \mu \mathrm{l}$ each of upstream and downstream primers, and $8 \mu \mathrm{l}$ RNase-free $\mathrm{H}_{2} \mathrm{O}$. Cycle parameters were as follows: $95^{\circ} \mathrm{C}$ for 30 seconds, followed by 45 cycles of $95^{\circ} \mathrm{C}$ for 5 seconds, $60^{\circ} \mathrm{C}$ for 30 seconds. This was followed by a melt curve reaction: $95^{\circ} \mathrm{C}$ for 15 seconds, then slowly heating from $55^{\circ} \mathrm{C}$ to $95^{\circ} \mathrm{C}$, for a total of 40 cycles. After the reactions were finished, the melting curves were generated.

\section{RESULTS}

\section{RNA Quality Control}

For pure RNA, the absorbance ratio $260 \mathrm{~nm} / 280 \mathrm{~nm}$ should be 1.8-2.1, and OD $260 \mathrm{~nm} / 230 \mathrm{~nm}$ should be $>1.8$ (Table I). When RNA samples are analyzed by gel electrophoresis, the $28 \mathrm{~S}$ and $18 \mathrm{~S}$ ribosomal RNA bands should be sharp and intense, with the upper band having about twice the intensity of the lower. Smaller, relatively diffuse bands may be present, arising from low molecular weight RNAs (tRNA and 5S ribosomal RNA). A diffuse smear is commonly seen between the $18 \mathrm{~S}$ and $28 \mathrm{~S}$ ribosomal bands and largely consists of mRNA and other heterogeneous RNA species. DNA contamination is evident as a high-molecular-weight smear, or a band migrating above the $28 \mathrm{~S}$ ribosomal RNA, as described by Zhang et al. (19). RNA degradation is indicated by smearing of the ribosomal RNA bands (Figure 1).

Chip data analysis and quantitative PCR found 48 miRNAs upregulated more than two fold in medulloblastoma, and 57 miRNAs downregulated more than two fold. The significant altered miRNAs are shown in Figure 2 and Table II.

\section{Largest Differences in Multiples of 20 miRNAs Bioinformatics Analysis}

Since the main function of miRNA is to regulate gene expression, we investigated the target genes of our identified miRNA in the Targetscan, microRNA.ORG and miRDBA databases. Enriched biological pathways were predicted according to the pathways in KEGG and BIOCARTA. The top ten pathways (sorted according to $p$ values) are shown in Table III. We found that the predicted target genes of hsamiR-647, hsa-miR-318, hsa-miR-208a-3p, hsa-miR-1207-5p, hsa-miR-1283 and hsa-miR-3202 were enriched in the Wnt signaling pathway, the MAPK signaling pathway and other pathways in cancer. 


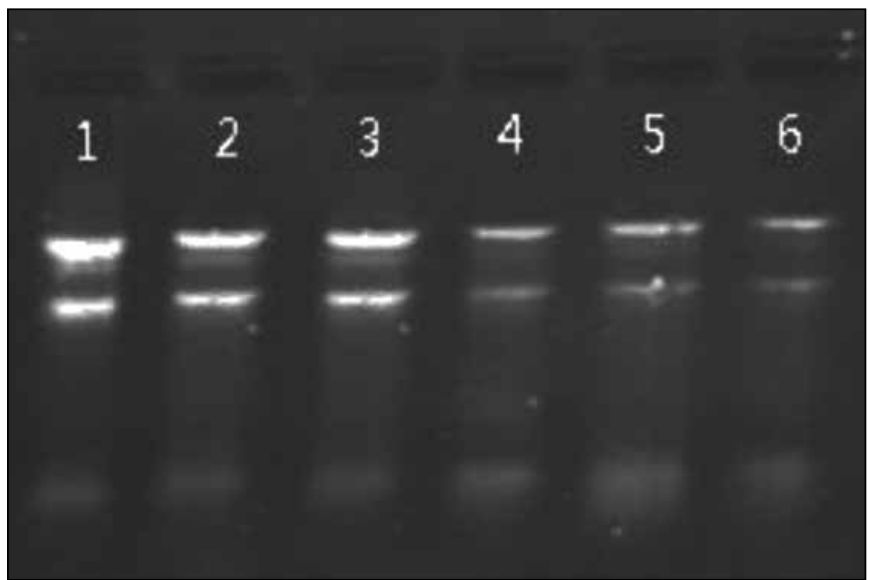

Figure 1: RNA Integrity and DNA contamination test by Denaturing Agarose Gel Electrophoresis.

\section{Real time Quantitative PCR}

In order to verify the reliability of the chip, we choose hsamiR-647, hsa-miR-3183, hsa-miR-208a-3P, hsa-miR-1207-5P, and hsa-miR-3202 for real time quantitative PCR validation; a sample amplification curve as shown in Figures 3A-E. HsamiR-208a-3p and hsa-miR-1207-5p were downregulated in cancer tissue, consistent with the results of the chip.

The $\Delta \mathrm{Ct}$ of hsa-miR-647 was significantly different between tumor samples and control samples $(t=3.068, p<0.05)$, an average of $2^{-\Delta \Delta \mathrm{Ct}}=2^{-1.483}=0.36$, was downregulated in cancer tissue, and was not consistent with the result of chip. The $\Delta \mathrm{Ct}$ of hsa-miR-3183 was significantly different between tumor samples and control samples $(\mathrm{t}=6.361, \mathrm{p}<0.05)$, an average of $2^{-\Delta \Delta \mathrm{Ct}}=2^{-3.01}=0.12$, was downregulated in cancer tissue, and was not consistent with the result of chip. The $\Delta \mathrm{Ct}$ of hsa-miR-208a-3p was significantly different between tumor

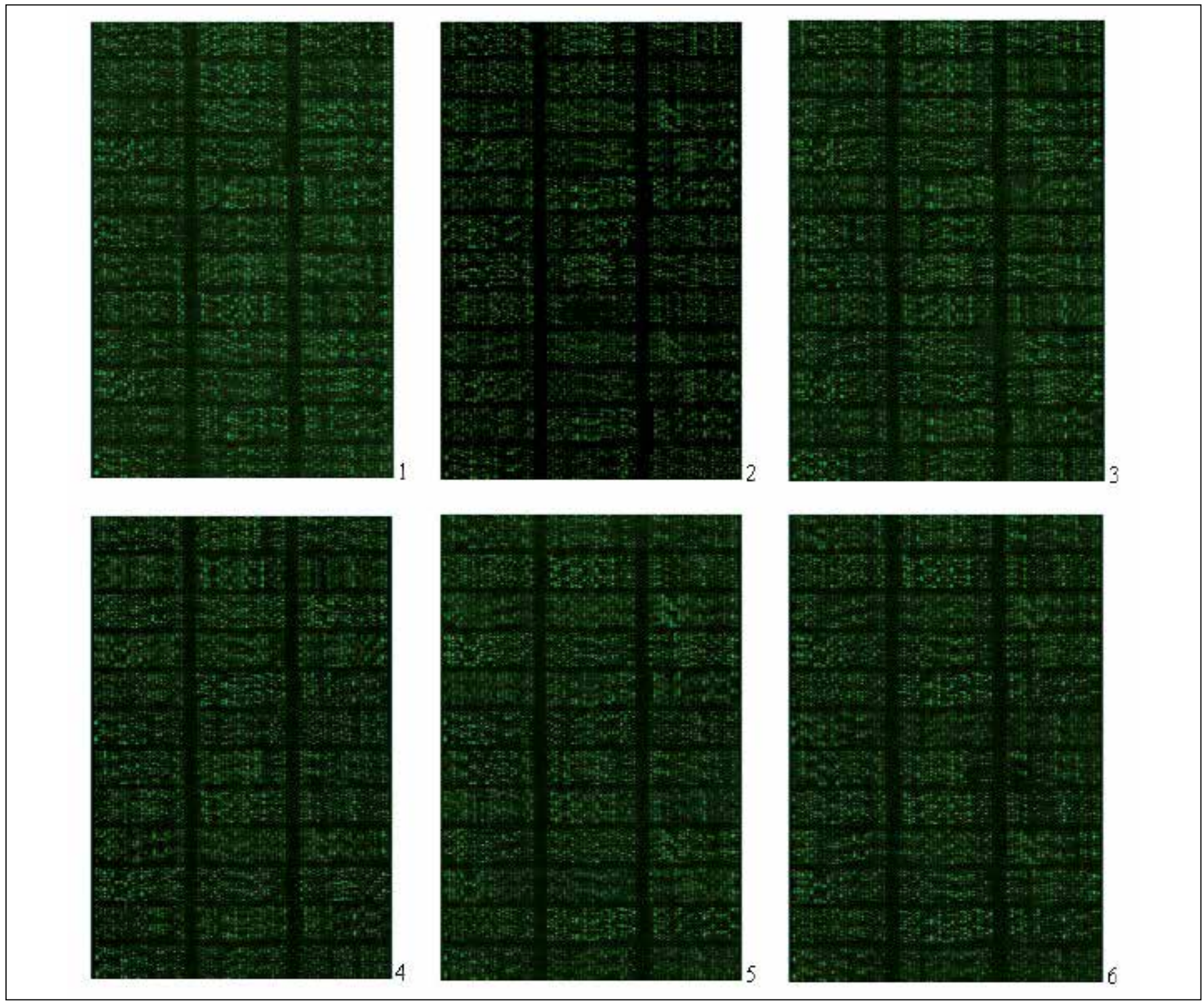

Figure 2: miRNA chip figures. Samples 1-3 were tumor tissue and Samples 4-6 were the corresponding negative controls. 
Table I: RNA Quantification and Quality Assurance by NanoDrop ND-1000

\begin{tabular}{cccccccc}
\hline Sample ID & OD260/280 Ratio & OD260/230 Ratio & Conc. $(\mathbf{n g} / \boldsymbol{\mu l})$ & Volume ( $\boldsymbol{\mu l})$ & Quantity (ng) & QC result pass or fail \\
\hline 1 & 2.01 & 2.36 & 2007.93 & 30 & 60237.90 & pass \\
\hline 2 & 1.97 & 1.95 & 974.77 & 10 & 9747.70 & pass \\
\hline 3 & 2.01 & 2.27 & 1713.17 & 40 & 68526.80 & pass & pass \\
\hline 4 & 1.98 & 2.29 & 827.37 & 20 & 16547.40 & 14819.00 & pass \\
\hline 5 & 1.97 & 2.06 & 1481.90 & 10 & 10 & 3310.60 & pass \\
\hline
\end{tabular}
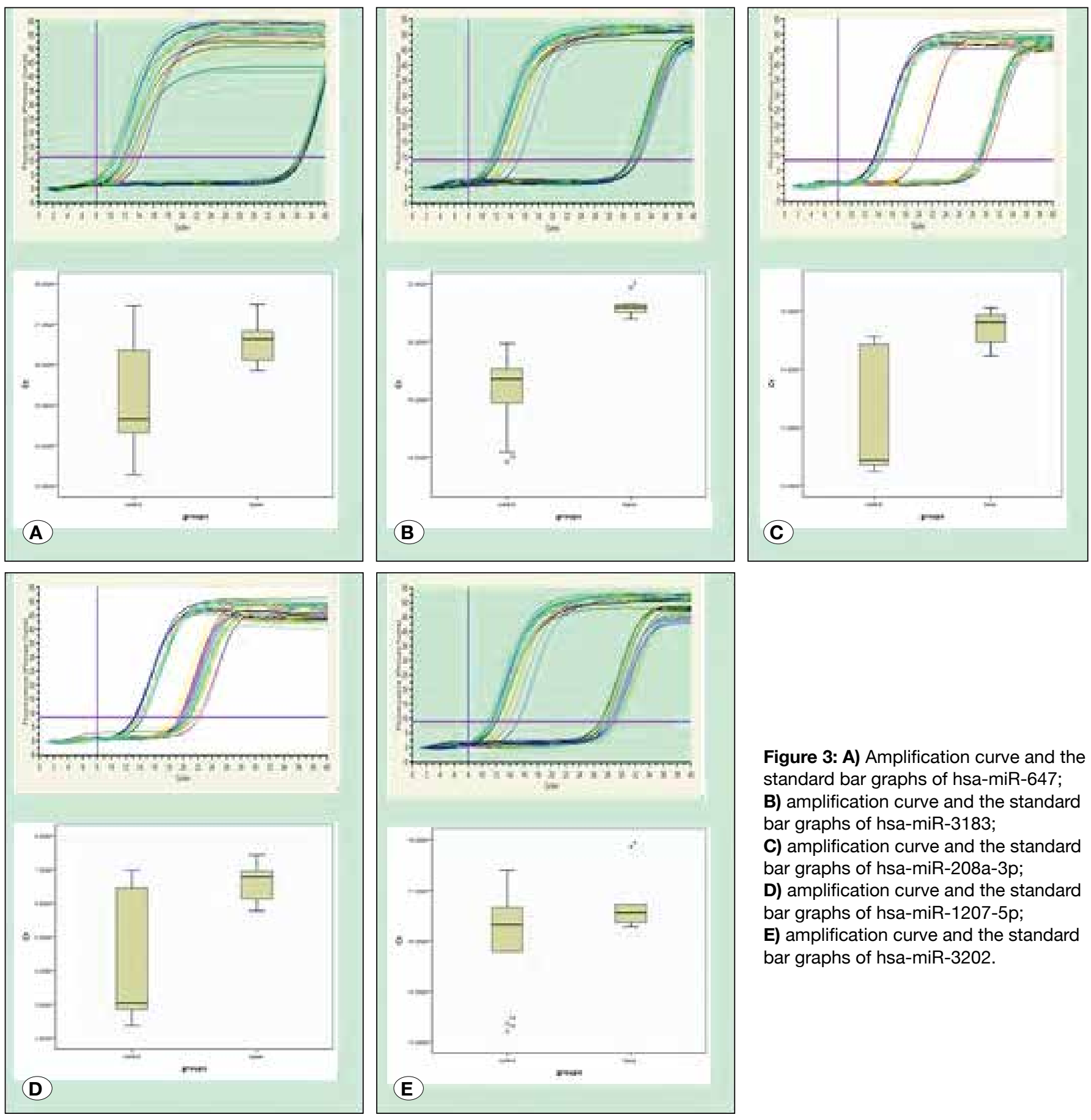

Figure 3: A) Amplification curve and the standard bar graphs of hsa-miR-647; B) amplification curve and the standard bar graphs of hsa-miR-3183;

C) amplification curve and the standard bar graphs of hsa-miR-208a-3p;

D) amplification curve and the standard bar graphs of hsa-miR-1207-5p;

E) amplification curve and the standard bar graphs of hsa-miR-3202. 
Table II: Significantly Differentially Expressed miRNAs

\begin{tabular}{|c|c|}
\hline miRNA s & multiple \\
\hline \multicolumn{2}{|l|}{ upregulated } \\
\hline hsa-miR-4518 & 7.49977 \\
\hline hsa-miR-3651 & 6.07914 \\
\hline hsa-miR-196a-3p & 4.14677 \\
\hline hsa-miR-5689 & 3.19520 \\
\hline hsa-miR-5096 & 3.78320 \\
\hline hsa-miR-647 & 2.14198 \\
\hline hsa-miR-3183 & 2.13916 \\
\hline \multicolumn{2}{|l|}{$\cdots$} \\
\hline \multicolumn{2}{|l|}{ downregulated } \\
\hline hsa-miR-138-5p & 0.07818 \\
\hline hsa-miR-5010-5p & 0.16658 \\
\hline hsa-miR-3665 & 0.19920 \\
\hline hsa-miR-208a-3p & 0.21806 \\
\hline hsa-miR-1283 & 0.23606 \\
\hline hsa-miR-1207-5p & 0.31042 \\
\hline hsa-miR-3202 & 0.38940 \\
\hline
\end{tabular}

samples and control samples $(t=4.384, p<0.05)$, an average of $2^{-\Delta \Delta C t}=2^{-3.266}=0.10$, was downregulated in cancer tissue, and was consistent with the result of chip. The $\Delta \mathrm{Ct}$ of hsa-miR1207-5p was significantly different between tumor samples and control samples $(\mathrm{t}=3.671, \mathrm{p}<0.05)$, an average of $2^{-\Delta \Delta \mathrm{Ct}}=$ $2^{-2.496}=0.18$, was downregulated in cancer tissue, and was consistent with the result of chip. The $\Delta \mathrm{Ct}$ of hsa-miR-3202 was not significantly different between tumor samples and control samples $(\mathrm{t}=1.650, \mathrm{p}>0.05)$, an average of $2^{-\Delta \Delta \mathrm{Ct}}=2^{-0.647}=$ 0.64 , had no obvious difference in expression between tumor tissue and tissue adjacent to a tumor, but was not consistent with the result of chip.

\section{DISCUSSION}

Studies have shown that miRNAs can serve as oncogenes or tumor suppressor genes in regulation of cellular proliferation and apoptosis (16), and play an important role in cancer. Using a combination of chip technology and real time quantitative PCR, we found for the first time that hsa-miR-208a-3p and hsa-miR-1207-5p expression was significantly downregulated in medulloblastoma. Liu et al. (13) reported that hsa-miR208a induced epithelial cells converted to mesenchymal cells, promoted the pancreatic cancer cell metastasis and invasion, the overexpression was concerned with the activation of AKT/GSK-3 $\beta /$ snail signaling pathway. Li et al. (12) have reported that hsa-miR-208a promoted the proliferation of human esophageal squamous cell carcinoma by inhibiting the expression of SOX6. Chen et al. (1) have reported that hsamiR-1207-5p inhibited the growth of gastric cancer cells by acting on telomerase reverse transcriptase. Our finding that the expressions of hsa-miR-208a-3p and hsa-miR-1207-5p were

Table III: Pathway Analysis Results

\begin{tabular}{|c|c|c|c|}
\hline Gene set name & $\begin{array}{l}\text { Number of } \\
\text { genes }\end{array}$ & $\begin{array}{l}\text { Significance } \\
\text { probability }\end{array}$ & Genes \\
\hline KEGG_WNT_SIGNALING_PATHWAY & 14 & 6.99E-07 & $\begin{array}{l}\text { NLK,CHP,PPP3R1, GSK3B, WNT1,CCND1,FZD5,FZD } \\
\text { 3,PPP2CA,CAMK2G,PPP2R5E,CSNK2A2,DKK2, VAN } \\
\text { GL2 }\end{array}$ \\
\hline KEGG_MAPK_SIGNALING_PATHWAY & 15 & 7.62E-05 & $\begin{array}{l}\text { NLK,CHP,PPP3R1,TGFBR1,STK4,FGF12,MEF2C,RAP } \\
\text { GEF2,MAP3K2,CACNA2D1,SRF,DUSP3,MAPK8IP3,D } \\
\text { USP16,TAOK2 }\end{array}$ \\
\hline KEGG_PATHWAYS_IN_CANCER & 16 & 1.37E-04 & $\begin{array}{l}\text { GSK3B, WNT1, CCND1,FZD5,FZD3, TGFBR1,STK4,FG } \\
\text { F12,PTCH1,SUFU,RXRA,RARA,IGF1,CCDC6,ARNT,E } \\
\text { TS1 }\end{array}$ \\
\hline KEGG_ENDOCYTOSIS & 11 & $7.15 \mathrm{E}-04$ & $\begin{array}{l}\text { SRC,SH3KBP1,SH3GLB2,SMURF1,RAB5B,ARF6,ARF } \\
\text { GAP3,AGAP1,VPS37A,EHD2,SMAP2 }\end{array}$ \\
\hline KEGG_MELANOGENESIS & 7 & $7.71 \mathrm{E}-03$ & GSK3B,WNT1,FZD5,FZD3,CAMK2G,GNAQ,CREB3L2 \\
\hline BIOCARTA_GABA_PATHWAY & 3 & $7.71 \mathrm{E}-03$ & $S R C, G A B R A 1, G A B R A 3$ \\
\hline BIOCARTA_P35ALZHEIMERS_PATHWAY & 3 & 8.59E-03 & GSK3B,PPP2CA,CDK5R1 \\
\hline BIOCARTA_GSK3_PATHWAY & 4 & 8.59E-03 & GSK3B,WNT1,CCND1,PPP2CA \\
\hline
\end{tabular}


downregulated in medulloblastoma suggests a role for these miRNAs in the tumor, but whether they are associated with tumor cell proliferation or invasion remains to be determined.

Given that hsa-miR-208a-3p and hsa-miR-1207-5p have largely unknown biological functions, we used bioinformatics methods to identify potential target genes and biological pathways. We used the Targetscan, microRNA.ORG and miRDBA databases to predict target genes. Hsa-miR-208a$3 p$ had eight target genes shared across the three databases, and hsa-miR-1207-5p had 81 target genes. Because of the complex regulation network of miRNAs, a miRNA could control multiple target genes, or one gene could be regulated by multiple miRNAs at the same time. Therefore, we focused on enriched biological pathways for the target genes to gain a broader understanding of miRNA function in medulloblastoma. Target genes were mainly enriched in the Wnt signaling pathway, MAPK signaling pathway and other cancer pathways, suggesting that the candidate target genes of hsa-miR-208a-3p and hsa-miR-1207-5p were closely involved in cancer's biological processes. Hence hsa-miR$208 a-3 p$ and hsa-miR-1207-5p were likely to control these genes and participate in the occurrence of medulloblastoma.

Particularly notable was the target gene enrichment in the Wnt signaling pathway, which plays an important role in a variety of biological processes, including cell proliferation and differentiation (14). Abnormal expression or activation of the Wnt pathway could lead to a variety of diseases and even cancer (11). $\beta$-catenin is the key member in the classic Wnt pathway (7). When Wnt protein binds to Fz transmembrane receptors, protein degradation is disrupted, and accumulated $\beta$-catenin moves to the nucleus and forms a complex with Tcf/Lef transcription factors to activate the expression of downstream target genes. Current research suggests that Wnt signal transduction pathways are closely associated with carcinogenesis of medulloblastoma (9).

This study showed that hsa-miR-208a-3p and hsa-miR $-1207-5 p$ expression in medulloblastoma was significantly downregulated, and target gene prediction showed that Nemo-like kinase (NLK), which participates in Wnt signaling, was a potential target gene of hsa-miR-208a-3p. NLK is an evolutionarily conserved serine/threonine kinase that suppressed the transcriptional activity of $\beta$-catenin/T-cell factor complex through phosphorylation of T-cell factor (10). The Wnt/ $\beta$-catenin signaling is thought to play a critical role in human carcinogenesis, so it is possible that NLK can act as a tumor suppressor by regulating the $\mathrm{Wnt} / \mathrm{\beta}$-catenin pathway. Cui et al.(3) found that NLK induced apoptosis in glioma cells via the activation of caspases, and suggested that NLK might be a useful independent prognostic indicator for glioma. Gene therapeutic approaches aimed at upregulating NLK expression could be developed for treatment of glioma. Wang et al.(17) found that miR-92b could regulate $W n t / \beta$-catenin signaling pathway by NLK, promoting cell proliferation and invasion of glioma.

The MAPK signaling pathway is another important pathway for extracellular signals to trigger reactions in the nuclei.
In 2001, MacDonald et al.(15) reported that in metastatic medulloblastoma, the Ras/MAPK (Ras proteins mediated MAPK activation) signaling pathway was upregulated; this was closely related to upregulation of the upstream gene platelet derived growth factor receptor alpha (PDGFRA). Previous research had shown that the activation of signaling pathways was related to gene mutation (18). But in 2006 Gilbertson et al.(6) suggested that the upregulation of the pathway in medulloblastoma might be caused by other mechanisms, through the sequencing of hot gene mutations in the pathway. This study showed that hsa-miR-208a-3p and hsa-miR-1207-5p expression in medulloblastoma was significantly downregulated, and target gene prediction showed that the NLK was potential target gene of hsa-miR208a-3p. RAPGEF2, CACNA2D1, DUSP3, MAPK8IP3 were identified as potential target genes of hsa-miR-1207-5p, all of which are involved in the MAPK signaling pathway. Zhang et al.(19) found that in in-vitro experiments, NEDD4-1 could regulate the migration and invasion of glioma cells through CNrasGEF ubiquitination.

\section{CONCLUSION}

Using bioinformatics methods to predict potential target genes for hsa-miR-208a-3p and hsa-miR-1207-5p narrowed the scope of our investigation to verify the real target genes. We found that downregulation of hsa-miR-208a-3p and hsa-miR-1207-5p might be involved in the occurrence of medulloblastoma through modulation of the Wnt and MAPK signaling pathways. Further research is needed to confirm this hypothesis and identify the key players in modulating medulloblastoma.

\section{REFERENCES}

1. Chen L, Lü MH, Zhang D, Hao NB, Fan YH, Wu YY, Wang SM, Xie R, Fang DC, Zhang H, Hu CJ, Yang SM: MiR-1207-5p and miR-1266 suppress gastric cancer growth and invasion by targeting telomerase reverse transcriptase. Cell Death Dis 5(1):e1034, 2014

2. Cohen A, Burgos-Aceves MA, Smith Y: Estrogen repression of microRNA as a potential cause of cancer. Biomed Pharmacother 78:234-238, 2016

3. Cui G, Li Z, Shao B, Zhao Y, Lu T, Wang J, Shi X, Wang J, Zuo G, Zhu W, Shen A: Clinical and biological significance of Nemo-like kinase expression in glioma. J Clin Neurosci 18(2):271-275, 2011

4. Fernandez-L A, Northcott PA, Taylor MD, Kenney AM: Normal and oncogenic roles for miRNAs in the developing brain. Cell Cycle 8(24):4049-4054, 2009

5. Ferretti E, De Smaele E, Po A, Di Marcotullio L, Tosi E, Espinola MS, Di Rocco C, Riccardi R, Giangaspero F, Farcomeni A, Nofroni I, Laneve P, Gioia U, Caffarelli E, Bozzoni I, Screpanti I, Gulino A: MicroRNA profiling in human medulloblastoma. Int J Cancer 124(3):568-577, 2009

6. Gilbertson RJ, Langdon JA, Hollander A, Hernan R, Hogg TL, Gajjar A, Fuller C, Clifford SC: Mutation analysis of PDGFRRAS/MAPK pathway activation in childhood medulloblastoma. Eur J Cancer 42(5): 646-649, 2006 
7. Guo F, Ren X, Dong Y, Hu X, Xu D, Zhou H, Meng F, Tian W, Zhao Y: Constitutive expression of PPARy inhibits proliferation and migration of gastric cancer cells and down-regulates Wnt/B-Catenin signaling pathway downstream target genes TERT and ENAH. Gene 584(1):31-37, 2016

8. Haijun Z, Yonghui Y, Jiake C, Hongjie D: Detection of the MicroRNA expression profile in skeletal muscles of burn trauma at the early stage in rats. Ulus Travma Acil Cerrahi Derg 21(4):241-247, 2015

9. He M, Li Y, Zhang L, Li L, Shen Y, Lin L, Zheng W, Chen L, Bian $X, N g$ HK, Tang L: Curcumin suppresses cell proliferation through inhibition of the $\mathrm{Wnt} / \beta$-catenin signaling pathway in medulloblastoma. Oncol Rep 32(1):173-180, 2014

10. Ishitani T, Kishida S, Hyodo-Miura J, Ueno N, Yasuda J, Waterman M, Shibuya H, Moon RT, Ninomiya-Tsuji J, Matsumoto K: The TAK1-NLK mitogen-activated protein kinase cascade functions in the $\mathrm{Wnt}-5 \mathrm{a} / \mathrm{Ca}(2+)$ pathway to antagonize Wnt/beta-catenin signaling. Mol Cell Biol 23(1):131-139, 2003

11. Kim SS, Cho HJ, Lee HY, Park JH, Noh CK, Shin SJ, Lee KM, Yoo BM, Lee KJ, Cho SW, Cheong JY: Genetic polymorphisms in the $W n t / \beta$-catenin pathway genes as predictors of tumor development and survival in patients with hepatitis $B$ virusassociated hepatocellular carcinoma. Clin Biochem 49(1011):792-801, 2016

12. Li H, Zheng D, Zhang B, Liu L, Ou J, Chen W, Xiong S, Gu Y, Yang J: Mir-208 promotes cell proliferation by repressing SOX6 expression in human esophageal squamous cell carcinoma. J Transl Med 12:196, 2014
13. Liu A, Shao C, Jin G, Liu R, Hao J, Song B, Ouyang L, Hu $X$ : MiR-208-induced epithelial to mesenchymal transition of pancreatic cancer cells promotes cell metastasis and invasion. Cell Biochem Biophys 69(2):341-346, 2014

14. Logan CY, Nusse R: The Wnt signaling pathway in development and disease. Annu Rev Cell Dev Biol 20:781-810, 2004

15. MacDonald TJ, Brown KM, LaFleur B, Peterson K, Lawlor C, Chen Y, Packer RJ, Cogen P, Stephan DA: Expression profiling of medulloblastoma: PDGFRA and the RAS /MAPK pathway as therapeutic targets for metastatic disease. Nat Genet 29(2): 143-152, 2001

16. Medina PP, Slack FJ: MiRNAs and cancer: An overview. Cell Cycle 7:2485-2492, 2008

17. Wang K, Wang X, Zou J, Zhang A, Wan Y, Pu P, Song Z, Qian C, Chen Y, Yang S, Wang Y: MiR-92b controls glioma proliferation and invasion through regulating $\mathrm{Wnt} /$ beta-catenin signaling via Nemo-like kinase. Neuro Oncol 15(5):578-588, 2013

18. Wetmore C: Sonic hedgehog in normal and neoplastic proliferation: Insight gained from human tumors and animal models. Curr Opin Genet Dev 13(1):34-42, 2003

19. Zhang H, Nie W, Zhang X, Zhang G, Li Z, Wu H, Shi Q, Chen Y, Ding Z, Zhou X, Yu R: NEDD4-1 Regulates migration and invasion of glioma cells through CNrasGEF Ubiquitination In Vitro. PLoS One 8(12): e82789, 2013 